\section{Remedios ancestrales como alternativa de tratamiento para infecciones respiratorias en tiempos de pandemia d}

\section{Ancestral remedies as alternative treat- ment for respiratory infections in pandemic times}

\section{Remédios ancestrais como alternativa de tratamento para ínfecções respiratórïas em tempos de pandemia}

\section{iD María Lorena Orellana Aguilar ${ }^{1}$} iD Ana María Mamani Rosas ${ }^{2}$

\section{Resumen}

Introducción: Las plantas medicinales fueron utilizadas con fines terapéuticos desde hace más de dos milenios antes de Cristo y actualmente se enfrentan a una pandemia y ha resurgido el interés por su uso principalmente para los síntomas respiratorios. El objetivo es determinar que remedios ancestrales fueron utilizados en tiempos de pandemia en la región metropolitana de Cochabamba. Metodología: El estudio es descriptivo, transversal y prospectivo, participaron 1520 sujetos de información a partir de 20 años. Resultados: Los remedios ancestrales fueron utilizados por el $53 \%$ de los sujetos, mientras que el $22 \%$ opto por fármacos, en cambio el $8 \%$ prefirió remedios medicinales y fármacos y el $17 \%$ indica ninguno. Los remedios son: Eucalipto $63 \%$, miel $50 \%$, Limón $48 \%$, Jengibre $42 \%$, Manzanilla $37 \%$, Ajo $30 \%$, Wira Wira $29 \%$, Cebolla $20 \%$, hierba luisa $9 \%$ y otros $32 \%$; la manifestación más frecuente para el uso fue la tos $44 \%$. La información la adquirieron en la familia $38 \%$, $21 \%$ internet, $8 \%$ vecinos, $9 \%$ medios de comunicación y $25 \%$ otros. Referente a la mejora de las manifestaciones el $52 \%$ indica haber tenido buenos resultados, $19 \%$ excelente, $13 \%$ regular, $2 \%$ mala y $15 \%$ reporta ninguno. Discusión: En la primera y segunda ola, se ha observado que los remedios naturales adquirieron mayor trascendencia, los centros comerciales y mercados brindan estos productos, por lo que el efecto curativo es notable. Por lo tanto, es necesario promover el desarrollo y fortalecimiento de políticas, programas, planes de protección, uso racional de la biodiversidad y la promoción de la medicina ancestral en Bolivia.

Palabras Claves: Plantas Medicinales, Usos Terapéuticos, Infecciones del Sistema Respiratorio.

\section{Summary}

Introduction: Medicinal plants were used in therapeutic purpos-
Correspondencia a:

${ }^{1}$ Médico Cirujano. Licenciada en Nutrición y Dietética. Centro de Investigación de Salud Pública UNITEPC. "CISPU".

Cochabamba - Bolivia.

${ }^{2}$ Licenciada en Enfermería. Investigadora del Centro de investigación de salud pública UNITEPC. "CISPU".

Cochabamba - Bolivia.

Email de contacto:

lorena72014@gmail.com anam.rosas1987@gmail. com

Procedencia y arbitraje:

No comisionado, sometido a arbitraje externo

Recibido para publicación: 20 de agosto del 2021

Aceptado para publicación: 30 de octubre del 2021

Citar como:

Orellana Aguilar ML, Mamani Rosas AM. Remedios ancestrales como alternativa de tratamiento para infecciones respiratorias en tiempos de pandemia. Recisa UNITEPC. 2021 ;8(2):53-60.

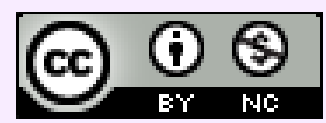

Esta obra está bajo una Licencia Creative Commons Atribución-NoComercial 4.0 Internacional.

Remedios ancestrales como alternativa de tratamiento para infecciones respiratorias en tiempos de pandemia. 
es since more than two millennials ago before Christ. Actually they face to pandemic and interest has resurfaced for the use of these medicinal plants, mainly for respiratory symptoms. The objective is to determinate which ancestral remedies were used in pandemic times in the metropolitan region of Cochabamba. Methodology: the study process is descriptive, transversal and prospective. 1520 subjects of information participated from 20 years old. Results: The ancestral remedies were used by the $53 \%$ subjects, while the $23 \%$ opted for drugs. But instead the $8 \%$ preferred medicinal remedies and the $17 \%$ indicates neither. The remedies are eucalyptus $63 \%$, honey $50 \%$, lemon $48 \%$, ginger $42 \%$, chamomile $37 \%$, garlic $30 \%$, wira wira $29 \%$, onion $20 \%$, Luisa herb $9 \%$ and others $32 \%$. The most frequent manifestation was used for the cough these remedies in $44 \%$. The information they acquired in the family $30 \%, 21$ $\%$ internet, $8 \%$ neighbors, $9 \%$ mass media and $25 \%$ others . Referring to the improve of clinical signs, the $52 \%$ indicate that they obtained good results, $19 \%$ excellent, 13 $\%$ regular, $2 \%$ bad and $15 \%$ reported none of these remedies. Discussion: In the first and second wave of Covid 19 it has been observed that natural remedies acquired grater importance. The shopping centers and markets provide these products, so that the healing effect is remarkable. Therefore it is necessary to promote the development and strengthening of politics, health programs, protection plans, rational use of biosafety and the promotion of ancestral medicine in Bolivia.

Keywords: Plants, Medicinal, Therapeutic Uses, Respiratory Tract Infections.

\section{Resumo}

Introdução: As plantas medicinais foram utilizadas com fins terapêuticos há mais de dois milênios antes de Cristo e, atualmente, para o enfrentamento da pandemia, ressurgiu o interesse pelo seu uso, principalmente, para os sintomas respiratórios. $\mathrm{O}$ objetivo deste trabalho foi determinar que remédios ancestrais foram utilizados em tempos de pandemia na região metropolitana de Cochabamba. Metodologia: Trata-se de um estudo descritivo, transversal e prospectivo. Participaram 1520 sujeitos de informação, a partir de 20 anos de idade. Resultados: Os remédios ancestrais foram utilizados por $53 \%$ dos sujeitos, enquanto que $22 \%$ optaram por medicamentos, em contrapartida, $8 \%$ preferiram tratamentos medicinais e fármacos ao mesmo tempo e 17\% indicaram o uso de nenhum tratamento. Os remédios utilizados foram: Eucalipto $(63 \%)$, mel $(50 \%)$, limão $(48 \%)$, gengibre $(42 \%)$, camomila $(37 \%)$, alho $(30 \%)$, wira $(29 \%)$, cebola $(20 \%)$, verbena $(9 \%)$ y outros $(32 \%)$. A informação foi adquirida através da família (38\%), internet (21\%), vizinhos ( $8 \%)$, meios de comunicação $(9 \%)$ e outros (25\%). Com relação à melhora das manifestações, $52 \%$ declararam haver tido bons resultados, $19 \%$, excelente, $13 \%$ regular, $2 \%$ ruim e $15 \%$ mencionaram nenhum resultado. Discussão: Nas primeira e segunda ondas, observaram-se que os remédios naturais adquiriram maior importância. Os centros comerciais e os mercados ofertaram estes produtos, pelo seu efeito curativo notável. Portanto, é necessário promover o desenvolvimento e o fortalecimento de políticas, programas, planos de proteção, o uso racional da biodiversidade e a promoção da medicina ancestral em Bolívia.

Palavras chaves: Plantas Medicinais, Usos Terapêuticos, Infecções Respiratórias.

\section{Introducción}

La medicina tradicional contempla una variedad de prácticas y terapias que son diferentes de un país a otro, es así que la Organización Mundial de la Salud (OMS). 
Determina una estrategia para el periodo 2014-2023 en el cual establece fortalecer el conocimiento, calidad, seguridad, eficacia y uso adecuado en las políticas nacionales. En ese sentido la ley 459 de Medicina Tradicional del 2013 en el artículo 1 hace referencia a Promover y fortalecer el ejercicio y la práctica de la medicina tradicional ancestral boliviana (1-4).

Asimismo, es considerada como: "un conjunto de conceptos, conocimientos, saberes y prácticas milenarias ancestrales, basadas en la utilización de recursos materiales y espirituales para la prevención y curación de las enfermedades, respetando la relación armónica entre las personas, familias y comunidad con la naturaleza y el cosmos, como parte del Sistema Nacional de Salud" (5).

Las plantas medicinales con fines curativos sé remota dos mil quinientos años antes de Cristo, en la actualidad frente a la pandemia, ha resurgido el interés de su uso, principalmente para sintomatología respiratoria (6-8).

De igual forma las plantas medicinales son aquellas que tienen propiedades de las cuales se puede extraer alguna o todas sus partes cuya finalidad es de aliviar, curar patologías, malestares físicos o anímicos. Las plantas siempre han sido una fuente común de medicamentos, administrándose como principio activo o en forma de preparaciones tradicionales. Por tanto, es prudente que los responsables de la toma de decisiones identifiquen plantas o extractos disponibles en las regiones que podrían agregarse de manera ventajosa a la lista nacional de medicamentos, o que incluso podrían sustituir algunas preparaciones farmacéuticas que deben de adquirirse o en todo caso importarse. (9-11).

Además, están comprobadas sus propiedades antibacterianas, antiinflamatorias y fungicidas, por lo que su consumo no está contraindicado. Sin embargo, estas plantas contribuyen a controlar algunos de los síntomas de los cuadros de infecciones respiratorias. No obstante, no hay evidencia de que en superficies tenga el impacto esperado de eliminar el virus. En ese sentido las medidas emanadas e implementadas por el ministerio de salud son importantes al momento de afrontar esta pandemia (12).

Por otro lado, según datos del Ministerio de Salud y Deportes en Bolivia se establece el indicador de riesgo de magnitud de propagación del virus COVID-19 y de acuerdo al decreto N. ${ }^{\circ} 4404$, los municipios deben de promover e implementar medidas preventivas y de contención.

El índice de Alerta Temprana calcula la tasa de incidencia de casos nuevos COVID-19 en la semana por cada 100.000 habitantes y está basado en los siguientes criterios:

\begin{tabular}{|c|c|c|}
\hline $\begin{array}{l}\text { Riesgo } \\
\text { Alto }\end{array}$ & $\begin{array}{l}\text { El contagio se encuentra desbordado, tiene lugar en ambien- } \\
\text { tes cerrados, espacios públicos, persona a persona en cual- } \\
\text { quier lugar, hogares y otros. Recomendándose medidas estric- } \\
\text { tas hasta desacelerar el contagio }\end{array}$ & $\begin{array}{l}\text { Mayor a } 57 \text { casos nuevos } \\
\text { COVID-19 por cada } 100 \\
\text { mil habitantes }\end{array}$ \\
\hline $\begin{array}{l}\text { Riesgo } \\
\text { Medio }\end{array}$ & $\begin{array}{l}\text { Hay un brote de foco propagado. La población se está con- } \\
\text { tagiando tanto en ambientes cerrados como en sus hogares, } \\
\text { el trabajo u otros. La población debe tomar en cuenta estos } \\
\text { factores y el riesgo de que se contagie es alto. }\end{array}$ & $\begin{array}{l}\text { Percentil } 75=57 \text { casos } \\
\text { nuevos COVID-19 por } \\
\text { cada } 100 \text { mil habitantes }\end{array}$ \\
\hline $\begin{array}{l}\text { Riesgo } \\
\text { Bajo }\end{array}$ & $\begin{array}{l}\text { Hay un brote de foco puntual. La población se estaría con- } \\
\text { tagiando en ambientes cerrados o espacios comunes como } \\
\text { cines, mercados, restaurantes, supermercados y otros. En- } \\
\text { contrar el foco puntual y bloquear el contagio, es la medida } \\
\text { recomendada. }\end{array}$ & $\begin{array}{l}\text { Percentil } 25=10 \text { casos } \\
\text { nuevos COVID-19 por } \\
\text { cada } 100 \text { mil habitantes }\end{array}$ \\
\hline
\end{tabular}

Remedios ancestrales como alternativa de tratamiento para infecciones respiratorias en tiempos de pandemia. 
Riesgo El virus está circulando en la población y hay riesgo de que las Inicial
Menor a 1 caso nuevo COVID-19 por cada 100 mil habitantes

Fuente: Ministerio de Salud y Deportes de Bolivia

La región metropolitana de Cochabamba está constituida por los municipios de Sipe Sipe, Vinto, Quillacollo, Colcapirhua, Tiquipaya, Cercado y Sacaba (13).

Los datos referentes al Índice de Alerta Temprana Según el Reporte N. ${ }^{\circ} 25$ de la semana epidemiológica 19 en Bolivia indica que existen, 100 municipios con Riesgo Alto, 57 municipios con Riesgo Medio, 29 municipios con Riesgo Bajo y 13 municipios con Riesgo Inicial. Además, los datos evidencian que Cochabamba se muestra entre los municipios con mayor riesgo después de Santa Cruz. Pero de acuerdo a las categorías Cochabamba tiene la mayor cantidad de municipios con riesgo Alto. Es así que los municipios con mayores casos se encuentran en el eje metropolitano (14).

En este contexto el presente trabajo de investigación tiene como objetivo determinar que remedios ancestrales fueron utilizados en tiempos de pandemia en la región metropolitana de Cochabamba.

\section{Metodología}

El estudio se desarrolló en el Marco del enfoque cuantitativo, es descriptivo, transversal y prospectivo. Participaron 1520 sujetos de información a partir de 20 años residentes en la región metropolitana del departamento de Cochabamba; los datos fueron recolectados por medio de un cuestionario en línea, del cual fueron descartados los sujetos que no respondieron a todas las preguntas.

Los datos fueron consolidados en una hoja Excel, posteriormente se desarrolló el análisis estadístico de frecuencias.

Los sujetos participaron voluntariamente previo consentimiento informado.

\section{Resultados}

Del trabajo participaron $58,7 \% n=892$ mujeres y $41,3 \% n=628$ hombres. El rango de edad corresponde a $51,8 \% n=788$ de $20-30$ años; $30,0 \% n=456$ entre $30-40$; asimismo $13,9 \% \mathrm{n}=212$ de $40-50$ y $4,2 \% \mathrm{n}=64$ más de 50 años.

En la tabla $\mathrm{N} .^{\circ} 1$ la cantidad de personas que recurrieron a los remedios ancestrales.

Tabla N.ํ 1 Población que recurrió a Plantas medicinales y medicamentos.

\begin{tabular}{l|l|l} 
Tratamiento & $\mathrm{N}^{\circ}$ & $\%$ \\
\hline Plantas Medicinales & 800 & $52,60 \%$ \\
\hline Medicamentos & 336 & $22,10 \%$ \\
\hline Plantas Medicinales y medicamentos & 120 & $7,90 \%$ \\
\hline Ninguno & 264 & $17,40 \%$ \\
\hline
\end{tabular}

En el grafico $\mathrm{N} .^{\circ} 1$ se observa la población que utilizo productos naturales; jengibre, limón, ajo, cebolla, hierba luisa, manzanilla, eucalipto, wira wira y miel, este último no es planta, pero fue nombrado por los encuestados. 
En el grafico $\mathrm{N} .^{\circ} 2$ se observa los síntomas y el 3 hace referencia a la fuente de información para usar estas plantas.

Gráfico №2 Sintomatología que fue intervenida con productos naturales durante la Pandemia

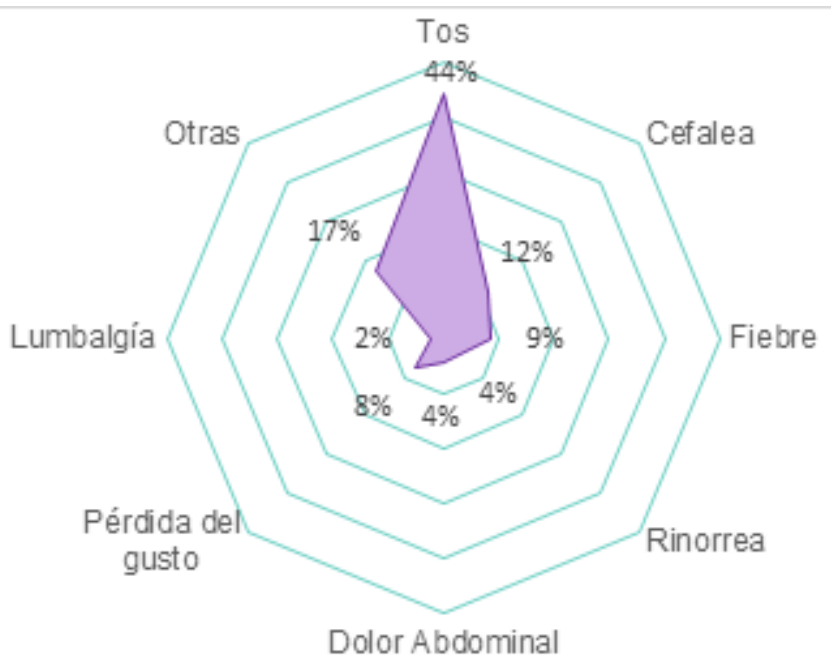

Gráfico N3 Fuente de información para la utilización de alternativas naturales

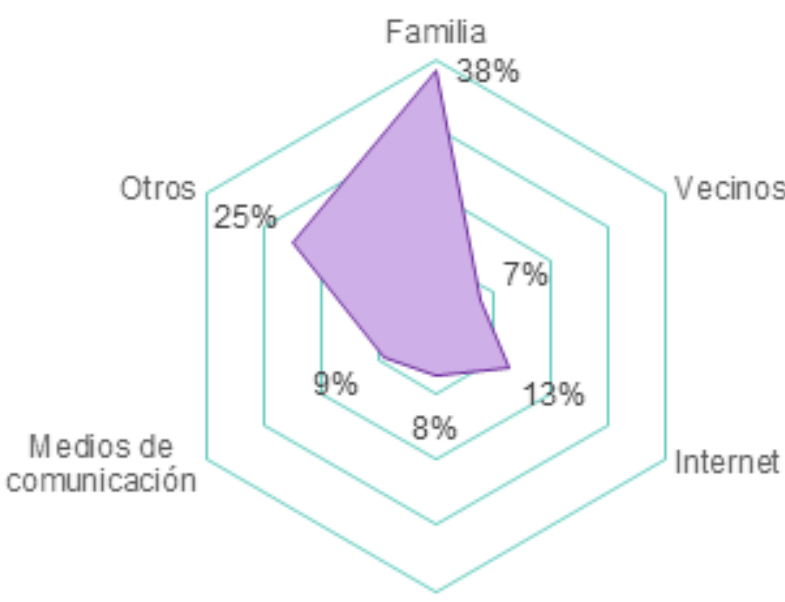

Redes sociales

Remedios ancestrales como alternativa de tratamiento para infecciones respiratorias en tiempos de pandemia. 
En el grafico $\mathrm{N} .^{\circ} 4$ se muestra los resultados que la población del eje metropolitano percibió con el uso de plantas naturales respecto al restablecimiento de salud, obteniéndose que el 19\% refiere haber tenido excelentes resultados, mientras que el $52 \%$ bueno, el $13 \%$ regular, mientras que el $2 \%$ mala y $15 \%$ indica ninguno.

Gráfico $N^{\circ} 4$ Resultados que perciben con el uso de plantas naturales respecto al restablecimiento de salud.

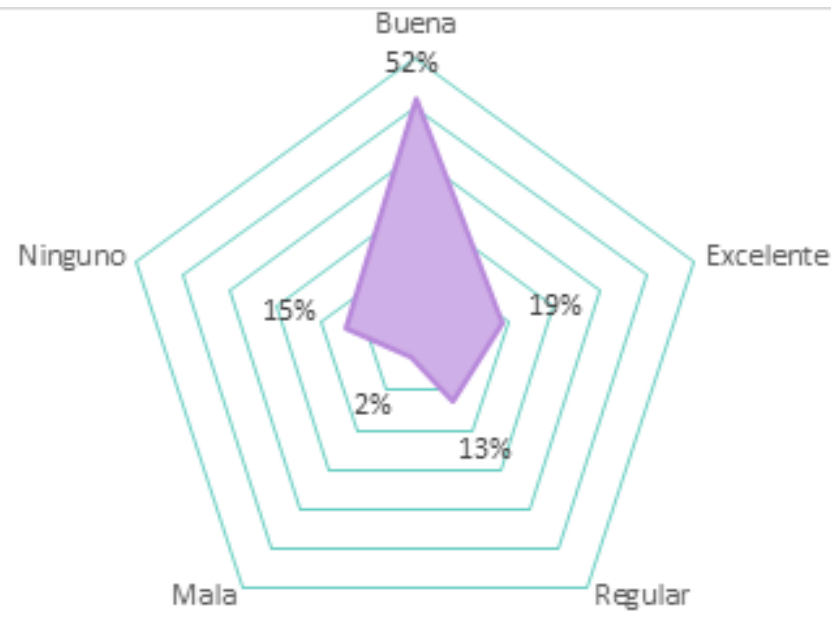

\section{Discusión}

En el entorno de la pandemia el estudio desarrollado evidencia que la población para poder prevenir o tratar ha buscado estrategias de afrontamiento, recurriendo a la medicina ancestral.

Según estimaciones de la Organización Mundial de la Salud, más del $80 \%$ de la población mundial, especialmente en los países en desarrollo, utiliza tratamientos tradicionales a base de plantas para sus necesidades de atención primaria de salud (15).

No obstante, en el eje metropolitano de Cochabamba se han empleado alternativas y una de ellas es revalorizar las plantas, productos naturales. Es así que el $53 \%$ de la población del eje metropolitano de Cochabamba utilizo esta alternativa para distintas afecciones. En nuestro medio durante la primera y segunda ola, se pudo observar que los productos naturales, cobraron mayor importancia y que los centros comerciales, mercados ofrecían dichos productos, con ello los efectos curativos resaltaron y se hicieron evidentes. Cabe señalar que además la sintomatología más prevalente fue la tos en las unidades de análisis, que el producto más utilizado fue el eucalipto, y que los medios de comunicación jugaron un rol esencial para que la población haga uso de estos productos.

Es fundamental promover el desarrollo y fortalecimiento de políticas, programas, planes de protección, uso racional de la biodiversidad y la promoción de la medicina ancestral en Bolivia. Algunos datos también hacen referencia a que en Bolivia se utilizó infusiones del eucalipto (Eucalyptus globulus), wira wira (Achyrocline alata, A. satureioides, A. venosa, Gnaphalium cheiranthifolium, Gamochaeta spp.) y manzanilla (Matricaria chamomilla). Las cuales son recomendaciones del Viceministerio de Medicina Tradicional e Interculturalidad, por las propiedades que presentan ante patologías respiratorias. 
Por lo tanto, es evidente que los medicamentos que derivan de las plantas están en espacios tanto de la medicina tradicional y moderna, en este sentido se debe de aunar esfuerzos por conservar la gran diversidad de especies vegetales que a futuro seguirán contribuyendo a mitigar el sufrimiento humano. Además, implementar tecnología para generar medicamentos a base de estas especies.

\section{Conflictos de Intereses}

Los autores declaramos no tener conflictos de interés para el presente estudio.

\section{Referencias Bibliográficas}

1. OMS. Progresos de la Asamblea Mundial de la Salud en lo concerniente a enfermedades no transmisibles y medicina tradicional [Internet]. 2014 [citado 4 de julio de 2021]. Disponible en: https://www.who.int/es/news/item/23-05-2014-world-health-assembly-progress-on-noncommunicable-diseases-and-traditional-medicine

2. Nigenda G, Mora-Flores G, Aldama-López S, Orozco-Núñez E. La práctica de la medicina tradicional en América Latina y el Caribe: el dilema entre regulación y tolerancia. Salud Pública México. 2001; 43(1):41-51.

3. Angulo-Bazán Y. Bibliometric indicators of peruvian scientific output about medicinal plants. Rev Peru Med Exp Salud Publica. 2 de diciembre de 2020;37(3):495503.

4. Pedraza-Alva G, Ramírez-Serrano CE, Pedraza F, Flores-Vallejo RDC, Villarreal ML, Pérez-Martínez L. From traditional remedies to cutting-edge medicine: Using ancient mesoamerican knowledge to address complex disorders relevant to psychoneuroimmunology. Brain Behav Immun. julio de 2019; 79:3-5.

5. Asamblea legislativa plurinacional de Bolivia. Ley de medicina tradicional ancestral boliviana. 459 dic 19, 2013 p. 26.

6. Gallegos-Zurita M. Las plantas medicinales: principal alternativa para el cuidado de la salud, en la población rural de Babahoyo, Ecuador. An Fac Med. 2016;77(4):32732.

7. Llivisaca-Contreras SA, Naranjo-Morán J, Pino-Acosta A, Pieters L, Vanden Berghe W, Manzano P, et al. Plants and Natural Products with Activity against Various Types of Coronaviruses: A Review with Focus on SARS-CoV-2. Mol Basel Switz. 5 de julio de 2021;26(13):4099.

8. Khare P, Sahu U, Pandey SC, Samant M. Current approaches for target-specific drug discovery using natural compounds against SARS-CoV-2 infection. Virus Res. diciembre de 2020; 290:198169.

9. Farnsworth NR, Akerele O, Bingel AS, Soejarto DD, Guo Z. Medicinal plants in therapy. Bull World Health Organ. 1985;63(6):965-81.

10. Signer J, Jonsdottir HR, Albrich WC, Strasser M, Züst R, Ryter S, et al. In vitro virucidal activity of Echinaforce ${ }^{\circledR}$, an Echinacea purpurea preparation, against coronaviruses, including common cold coronavirus 229E and SARS-CoV-2. Virol J. 9 de septiembre de 2020;17(1):136.

11. Stan D, Enciu A-M, Mateescu AL, Ion AC, Brezeanu AC, Stan D, et al. Natural Compounds With Antimicrobial and Antiviral Effect and Nanocarriers Used for Their 
Transportation. Front Pharmacol. 2021; 12:723233.

12. Maldonado C, Paniagua-Zambrana N, Bussmann RW, Zenteno-Ruiz FS, Fuentes AF. La importancia de las plantas medicinales, su taxonomía y la búsqueda de la cura a la enfermedad que causa el coronavirus (COVID-19). Ecol En Boliv. 2020;55(1):1-5.

13. Manzano N. El complejo territorial de la región metropolitana de Cochabamba. 1a ed. Cochabamba: IESE - UMSS; 2015.

14. Ministerio de Salud y Deportes de Bolivia. Índice de Alerta Temprana Reporte 25 [Internet]. 2021 [citado 5 de julio de 2021]. Disponible en: https://www.minsalud. gob.bo/component/jdownloads $/$ ?task=download. send $\&$ id $=632 \&$ catid $=29 \& m=0 \& l-$ temid $=646$

15. Rojas Ochoa F, Silva Ayçaguer LC, Sansó Soberats FJ, Alonso Galbán P. El debate sobre la Medicina Natural y Tradicional y sus implicaciones para la salud pública. Rev Cuba Salud Pública. 2013;39(1):107-23. 